\title{
A educação nutricional nos programas oficiais de prevenção da deficiência da vitamina A no Brasil ${ }^{1}$
}

\author{
Nutrition education in the official programs for \\ preventing vitamin A deficiency in Brazil
}

Lívia Penna Firme RODRIGUES²

Maria José RONCADA ${ }^{3}$

RE S U M O

O objetivo deste artigo é identificar a inserção da Educação Nutricional nos programas oficiais brasileiros de combate à deficiência de vitamina A no Brasil, no período de 1968 a 2008. A metodologia utilizada foi pesquisa bibliográfica e pesquisa documental, onde foram analisados documentos oficiais, arquivados na Coordenação Geral de Políticas de Alimentação e Nutrição, do Ministério da Saúde, referentes aos programas governamentais nacionais para a prevenção e controle da Deficiência de Vitamina A. Descreve-se a trajetória da Educação Nutricional nos programas oficiais de combate à Deficiência de Vitamina A no Brasil, desde sua implantação até os dias atuais, constatando-se que a prática de Educação Nutricional é recomendada, porém não executada. Isso ocorre porque as ações propostas nos programas oficiais são sugeridas como ações pontuais, ao invés de estarem inseridas em uma política pública de promoção da saúde. Nesse sentido, o atual programa de prevenção e combate a essa Deficiência evoluiu, pois recomenda o aleitamento materno e a alimentação saudável - medidas de promoção da saúde -, como essenciais para a sua prevenção. Conclui-se que, à medida que a Educação Nutricional avance e se estabeleça como parte de uma política pública intersetorial de promoção para a saúde, a alimentação saudável passe a fazer parte da vida dos brasileiros, prevenindo não apenas a Deficiência de Vitamina A, mas, também, outras doenças carenciais e as crônicas não transmissíveis.

Termos de indexação: Deficiência de vitamina A. Educação alimentar e nutricional. Promoção da saúde.

A B S T R A C T

The objective of this article is to identify the insertion of nutrition education in the official Brazilian programs for the prevention of vitamin A deficiency in Brazil, in the period from 1968 to 2008. The employed methodology

\footnotetext{
${ }^{1}$ Artigo elaborado a partir da tese de L.P.F. RODRIGUES, intitulada "A Educação nutricional no Brasil: contribuição para a prevenção da deficiência de vitamina A”. Universidade de Brasília; 2009.

2 Universidade de Brasília, Faculdade de Planaltina. Vl. Nossa Senhora de Fátima, 73300-000, Planaltina, DF, Brasil. Correspondência para/Correspondence to: L.P.F. RODRIGUES.E-mails: <liviapennafirme@gmail.com>; <liviapenna@unb.br>.

3 Universidade de Brasília, Faculdade de Ciências da Saúde. Brasília, DF, Brasil.
} 
298 | L.P.F. RODRIGUES \& M.J. RONCADA

was a literature and document research, analyzing the official documents kept by the General Coordination of Food and Nutrition Policies of the Ministry of Health regarding national governmental programs for the prevention and control of vitamin A deficiency. The trajectory of nutrition education in the official programs for the prevention of Vitamin A deficiency in Brazil is described, from its implementation to the present days. Nutrition education was found to be recommended but not done. This happens because the actions proposed by the official programs are solitary actions when in fact they should be inserted in a health-promoting public policy. In this sense, the current program for the prevention of vitamin A deficiency has improved, since it recommends breastfeeding and healthy eating habits as essential factors for its prevention, measures that promote health. In conclusion, as nutrition education advances and is established as part of a trans-sector health-promoting public policy, healthy eating habits become part of Brazilians' daily life, preventing not only vitamin A deficiency, but also other nutritional deficiencies and non-communicable chronic diseases.

Indexing terms: Vitamin A deficiency. Food and nutrition education. Health promotion.

\section{N T R O D U ÇÃ O}

A Deficiência de Vitamina A (DVA) é um dos problemas nutricionais mais importantes e frequentes no mundo, presente em mais de cem países, de grande impacto social e, ao mesmo tempo, de mais fácil prevenção. Tem alta prevalência em regiões da Ásia, África e América Latina em populações de baixo nível socioeconômico e condições sanitárias insatisfatórias. Cerca de cinco a dez milhões de crianças por ano apresentam deficiência subclínica; 250 milhões apresentam sinais clínicos e bioquímicos, sendo mais gravemente atingidas, com registros de 250 mil a 500 mil novos casos de cegueira por ano'.

O Brasil não dispõe de um inquérito em nível nacional sobre a prevalência da DVA, porém foram realizadas várias pesquisas nos últimos quarenta anos que utilizaram indicadores clínicos, bioquímicos e/ou dietéticos, cujos resultados estão agrupados e comentados em trabalhos de revisão sobre a situação da DVA no país ${ }^{2-7}$. Segundo publicação recente intitulada "Carências de Micronutrientes"8 a DVA é problema de saúde pública no Brasil, um dos países mais afetados na América, atingindo principalmente as crianças em idade pré-escolar. No período de 1995 a 2001, a DVA foi responsável pelo óbito de 36,6 mil crianças brasileiras.

O Programa Nacional de Suplementação de Vitamina $A^{9}$ afirma que as principais causas da DVA no País estão relacionadas à falta de amamentação ou desmame precoce, consumo insuficiente de alimentos ricos em vitamina $A$, consumo inadequado, nas famílias de baixa renda, de alimentos que contêm gordura, e infecções frequentes, embora vários desses aspectos possam ser modificados e melhorados por meio da Educação Nutricional (EN). Apesar da sua importância, o que se observa nos programas de combate à DVA, no Brasil, é que a EN é sugerida como ação de longo prazo, não tendo o mesmo peso, divulgação e investimento destinado a outras medidas, como o enriquecimento ou fortificação de alimentos e a distribuição periódica de suplementos de vitamina A, consideradas como estratégias de médio e curto prazos, respectivamente, e que têm sido executadas, mesmo que parcialmente.

Destaca-se a importância da alimentação para a prevenção dessa deficiência, considerando-se que as principais fontes de origem animal de vitamina A na forma de seus ésteres, resumem-se a algumas espécies de pescados, fígados de boi e de frango, ovos, queijos (preparados com leite integral), manteiga e leite integral, alimentos comuns e frequentes no comportamento alimentar do povo brasileiro, desde que haja segurança alimentar. As fontes mais importantes de vitamina A, por serem de baixo custo e, portanto, mais acessíveis, são os alimentos ricos em carotenóides pró-vitamina A (principalmente o beta-caroteno), abundantes no Brasil, de fácil aquisição e de rápido preparo, pois muitos deles podem ser consumidos in natura. Os alimentos ricos em betacaroteno incluem legumes e frutas de cor amarelo-alaranjada (cenoura, abóbora madura, moranga, manga, mamão), as verduras de cor verde-escura (bredo, caruru, mostarda, couve) e os frutos de 
palmáceas, como dendê, buriti e pupunha ${ }^{10}$. No entanto, muitos deles têm consumo reduzido devido a questões culturais, o que coloca a EN como estratégia importante na prevenção e combate à DVA no Brasil.

Considerando a importância do tema "educação nutricional como estratégia de combate à deficiência de vitamina $A$ ", o objetivo deste artigo é identificar a inserção da EN nos programas oficiais brasileiros de combate à DVA, no período de 1968 a 2008, e analisar a trajetória da EN no mesmo período, propondo ações de EN possíveis para a prevenção de DVA no Brasil.

\section{MÉ T O D O S}

A metodologia utilizada foi a pesquisa bibliográfica ${ }^{11}$, desenvolvida com base em material impresso e virtual já publicado, como livros, outras publicações relevantes e artigos científicos, encontrados nas bases de dados Medline, PubMed e SciELO, Lilacs, em que se utilizaram as palavras-chave "deficiência de vitamina A" e "educação nutricional".

Para identificar a inserção de EN nos programas oficiais brasileiros de combate à DVA no período de 1968 a 2008, utilizou-se a pesquisa documental ${ }^{11}$ onde foram analisados documentos oficiais, arquivados na Coordenação Geral de Políticas de Alimentação e Nutrição (CGPAN) do Ministério da Saúde, referente aos programas governamentais nacionais para a prevenção e controle da DVA, elaborados no período de 1968 a 2008. A partir de visitas a esse órgão, foram acessados os poucos documentos existentes, considerando-se que vários deles extraviaram-se durante o processo de extinção do Instituto Nacional de Alimentação e Nutrição (INAN). Para atenuar essa lacuna, contou-se com a colaboração de funcionário público, Gestor do Programa Nacional da DVA por muitos anos, que prestou depoimento gravado e analisado posteriormente. Analisaram-se, nos documentos encontrados, as ações de EN propostas, sua execução e avaliação.

\section{RESULTADOS E DISCUSSÃO}

\section{Histórico das ações de educação nutricional no controle da DVA no Brasil}

Ao se descrever a trajetória da EN nos programas de combate à DVA no Brasil, observa-se uma lacuna permanente nas ações de EN realizadas, o que se repete ao longo dos últimos quarenta anos, quando os programas de prevenção dessa deficiência tiveram início com a criação, em 1972, do Instituto Nacional de Alimentação e Nutrição (INAN).

Foi a partir dos anos setenta que o governo federal estabeleceu as primeiras ações de prevenção, diagnóstico e combate à DVA, com uma abordagem ampla, visando a um contexto de ações para a sua erradicação total'12. Em 1977, o Ministério da Saúde promoveu, por meio do INAN, o Seminário sobre Hipovitaminose A no Brasil, realizado em Recife, no período de 27 a 29 de setembro, reunindo os especialistas nacionais do assunto, pela primeira vez, com o objetivo de avaliar a situação da DVA no Brasil e estabelecer medidas para seu controle ${ }^{13}$.

Nessa época, houve uma decisão política favorável ao combate das carências nutricionais, entre elas a DVA, devido à criação do PRONAN - Programa Nacional de Alimentação e Nutrição - e, também, com o comprometimento do Brasil e de outros países com o Plano Decenal de Saúde para as Américas, que tinha como meta a redução em $30 \%$ da prevalência anual da DVA, até o ano de $1982^{12}$, que não foi atingida.

A partir da elaboração de um documento básico para aquele Seminário, constatou-se a gravidade da DVA no Brasil, exigindo ações emergenciais de curto prazo nas regiões afetadas. Em 1979, o INAN elaborou proposta para distribuição de megadoses de vitamina $A$, que não foi colocada em prática, pois, embora houvesse urgência devido à grave incidência de DVA em algumas regiões, havia, também, insuficiência de dados epidemiológicos que justificassem essa medida no 
País como um todo, segundo os documentos oficiais ${ }^{13,14}$. Uma explicação para isso é que os dados apresentados, por serem regionais, não foram suficientes para convencer o governo, na época, da gravidade da situação e da necessidade de se iniciar um programa de abrangência nacional.

Um levantamento epidemiológico mais amplo, elaborado pelo INAN em março de 1981, levou à elaboração do projeto preliminar intitulado "Projeto de combate à hipovitaminose $\mathrm{A}$ no Brasil"15, com a proposta de prevenir e combater essa deficiência - dando atenção especial para a xeroftalmia em nutrizes e pré-escolares -, a partir de 1983, quando foram iniciadas as primeiras atividades de controle da DVA no país, com a suplementação de megadoses de vitamina $A$. Desde então, a EN foi citada e reconhecida oficialmente como uma ação importante na prevenção da DVA.

Sugeriu-se a promoção da educação alimentar em áreas identificadas como de alto risco, enfatizando o estímulo ao consumo de alimentos ricos em retinol e carotenóides, de produção local, a partir da implantação de hortas, além do estímulo ao aleitamento materno ${ }^{15}$. No entanto, essas medidas não foram colocadas em prática no país como um todo. Não há documentos técnicos relativos à implantação e avaliações de programas de EN dessa época e, segundo depoimento prestado por Gestor do Programa Nacional de Combate à DVA, não cabia ao INAN responsabilizar-se pela execução direta das ações de EN, pois a principal função desse órgão era garantir a distribuição de vitamina A nas áreas de risco, a partir da sua doação por organismos internacionais. Mesmo assim, o INAN recomendava às secretarias estaduais e municipais de saúde planejar e executar as ações educativas, que não ocorriam, devido à desinformação local sobre a gravidade da DVA. A falta de apoio, por parte dos gestores locais e das Secretarias Estaduais de Saúde para essas iniciativas, era devida aos vários problemas operacionais existentes com a distribuição das megadoses de vitamina $A$, que consumiam o tempo disponível.
O Gestor do programa acrescentou, ainda, que o desinteresse em prevenir a DVA nas comunidades era consequência do desconhecimento sobre a importância da prevenção dessa deficiência, permitindo que outras enfermidades como a paralisia ou a AIDS chamassem mais a atenção dos profissionais de saúde e da comunidade. Mesmo assim, houve algumas iniciativas isoladas, também não avaliadas, de alguns Estados do Brasil que, mais interessados, elaboraram cartilhas, cartazes e manuais para profissionais de saúde, alertando sobre os riscos da DVA e a importância da prevenção, medidas que têm uma relevância em termos de informação, mas não podem ser consideradas ações de EN.

Em 1986, a Reunião Técnica Conjunta entre os poucos pesquisadores pioneiros nacionais com o Grupo Consultivo Internacional de Vitamina A (IVACG), em Brasília, DF, levou à criação do Grupo Consultivo Nacional para Hipovitaminose A, chamado de Grupo Brasileiro de Vitamina A (GBVA), cujo objetivo era o de assessorar o INAN nas intervenções e pesquisas necessárias. Esse Grupo sugeriu, para o controle da DVA no Brasil, além da intervenção com doses maciças nas regiões de alta prevalência, ações básicas como a inclusão de, pelo menos, um alimento rico em vitamina A nos programas oficiais e o controle dos preços desses alimentos, além da proibição de distribuição do leite desengordurado9. Desconhece-se até que ponto essas ações foram colocadas em prática e avaliadas.

Em 1988, o INAN decidiu introduzir alguns alimentos formulados no Programa de Suplementação Alimentar (PSA), cujas especificações nutricionais atendessem a um quarto das necessidades diárias da população-alvo, devendo o formulado lácteo conter $3.000 \mathrm{UI} / \mathrm{l}$ de vitamina $\mathrm{A}^{16}$. Um ano depois, em 1989, houve a recomendação para a adição de quantidades adequadas de vitamina A ao leite industrializado usado no Brasil, com o objetivo de reforçar o seu valor nutritivo e melhorar o estado nutricional da população. Embora essas medidas se refiram ao consumo do leite e formulados lácteos enriquecidos - aspectos 
que deveriam ser divulgados ao público consumidor -, não se sabe se houve ações e/ou informações nesse sentido, o que mostra como a EN não era valorizada como um instrumento capaz de trazer mudanças nos hábitos alimentares da população.

Durante o período de 1983 a 1993, a distribuição das megadoses de vitamina A ocorreu de forma descontínua, com grandes flutuações na cobertura, tornando a sua distribuição irregular em várias regiões do país ${ }^{17}$. A partir de 1994, o Ministério da Saúde criou o Programa Nacional de Controle da Deficiência de Vitamina A, a ser gerenciado pelo INAN, o que permitiu a expansão e manutenção da regularidade na distribuição das megadoses de vitamina $A$, atingindo cerca de 1,5 milhões de beneficiários. Como recurso de EN, o Ministério da Saúde, INAN e Coordenação de Saúde Materna Infantil (COMIN), elaboraram a cartilha intitulada "Conheça a Hipovitaminose A e Aprenda a Preveni-la" 18 destinada às mães e agentes de saúde das comunidades atendidas pela distribuição de megadoses de vitamina A. O conteúdo disponibilizava informações à população para o consumo de alimentos ricos em retinol e carotenóides na composição de sua alimentação diária.

Tradicionalmente, esse tipo de material não atinge a população alvo, devido à tiragem limitada ocasionada por seu alto custo. A leitura do conteúdo dessas cartilhas, mesmo estando em linguagem acessível, não é suficiente para convencer as pessoas a consumirem os alimentos indicados, devendo-se, ainda, considerar que parte da população não tem condições de compreender o que lê ou é analfabeta. Ou seja, a cartilha é um recurso que pode beneficiar a prática da EN quando praticada dentro de um contexto de ações; no entanto, de forma isolada, pode apenas ajudar a informar as mães e agentes comunitários de saúde que a ela tiverem acesso, com o risco de ter uma vida útil bastante reduzida.

Em 1997, com o processo de extinção do INAN, o programa foi paralisado, tendo sido retomado, em 1999, pela Coordenação Geral da Polí- tica de Alimentação e Nutrição (CGPAN) do Ministério da Saúde, que passou a ter responsabilidade pela elaboração, execução e avaliação das ações de alimentação e nutrição desenvolvidas no país. O programa é executado nas secretarias estaduais e municipais de saúde, bem como nas coordenações estaduais de alimentação e nutrição, com o apoio do Fundo das Nações Unidas para a Infância (UNICEF) e da Organização Pan-Americana de Saúde (OPAS) ${ }^{17}$.

No período de 2002 e 2003, a partir dos dados publicados pela Pesquisa Nacional de Micro-Nutrientes, a cobertura de suplementação de vitamina A foi ampliada para outras regiões consideradas de risco no Brasil. Iniciou-se a administração de megadoses em puérperas, no período pós-parto imediato, garantindo-se o aporte de vitamina A, por meio do leite materno, para as crianças menores de seis meses de idade, que estivessem sendo amamentadas ${ }^{19}$. Não há referências sobre medidas educativas que esclarecessem as mulheres da importância de receber esse aporte de vitamina A no pós-parto, o que poderia ser um estímulo para a continuidade do aleitamento, considerando-se que o recém-nascido estaria mais protegido da DVA e se estaria valorizando a participação ativa da mãe nesse processo.

Atualmente, permanece em vigor o Programa Nacional de Suplementação de Vitamina A ("Vitamina A Mais"), que continua com a estratégia de suplementação com megadoses de vitamina A, como medida de curto prazo, para o controle da deficiência desse nutriente. Como estratégia de prevenção indica-se a promoção do aleitamento materno exclusivo até o $6^{\circ}$ mês, ocasião em que se inicia a introdução de alimentação complementar, mas mantendo o aleitamento até dois anos de idade, pelo menos; e a promoção da alimentação saudável, fortemente recomendada, para assegurar informações necessárias ao incentivo do consumo de alimentos ricos em vitamina A pela população ${ }^{20}$.

A estratégia de distribuição de megadoses de vitamina $A$, integrada ao Programa Nacional 
de Imunização, tornou o Brasil pioneiro nessa iniciativa, que passou a ser posteriormente preconizada pela Organização Mundial de Saúde em vários países, como medida eficiente de combate da DVA ${ }^{17}$. Por outro lado, uma avaliação recente do atual Programa Nacional de Suplementação de Vitamina A ("Vitamina A Mais") realizada no estado da Bahia, no período de 1999 a $2002^{21}$ mostrou que a realização de atividades educativas, previstas a longo prazo, e que deveriam ser executadas pelos gestores locais, visando ao aumento do consumo de alimentos ricos em vitamina $A$, não aconteceu e/ou não produziu os resultados esperados. Das 1213 famílias entrevistadas, 74,6\% mostraram não possuir nenhum conhecimento sobre vitamina A e, segundo os gestores, isso se deu devido à limitação de tempo disponível e à falta de recursos humanos, mostrando que as ações educativas, no transcorrer dos dias nacionais de vacinação, não são recomendadas. Essa pesquisa deixa clara a necessidade de serem criadas novas estratégias para desenvolver a EN de forma permanente e por pessoal treinado nos programas oficiais de prevenção e combate da DVA.

\section{A TRAJETÓRIA DA EDUCAÇÃ O NUTRICIONAL NO BRASIL E NOS PROGRAMAS OFICIAIS DE PREVENÇÃO DA D V A}

A EN é uma ação de promoção à saúde e pertence ao âmbito da Educação para a Saúde estando, portanto, exposta às suas influências históricas e sociais. A Educação para a Saúde pode ser descrita como um conjunto de saberes e práticas, produzidos cientificamente, transmitidos à população por profissionais de saúde, com o objetivo de evitar doenças e promover a saúde ${ }^{22}$.

Fazendo-se um paralelo entre a trajetória da Educação para a Saúde e a EN, observam-se aspectos comuns. Resumindo-se o histórico da EN ${ }^{23}$, tem-se que o seu interesse acadêmico remonta à década de quarenta, em virtude do pós-guerra. Na mesma época, no Brasil, surgiu uma profissional de saúde intitulada "Visitadora de Alimentação", que ia aos domicílios fazer educação alimentar de forma tradicional, de acordo com a Educação para a Saúde realizada na época que, de forma autoritária, ditava os preceitos alimentares a serem seguidos e que foi considerada invasiva pela população. Nos anos 1960 e 1970, a EN abandonou suas raízes sociais e antropológicas e, influenciada pela área médica, teve, como objetivo, a mudança de comportamento alimentar baseada no modelo biologicista, que determinava as normas para se alimentar corretamente, independentemente dos fatores sociais envolvidos. $\mathrm{E}$, posteriormente, nas décadas de 1970 e 1980, seguindo a tendência mundial, a EN foi focada na mudança de hábitos ${ }^{23}$.

A filosofia proposta pela Educação Popular e que passou a ser utilizada na Educação para a Saúde no Brasil, a partir da década de 1970, não foi assimilada na prática da EN, exceto em alguns setores progressistas, pois dominava o modelo tradicional, biologicista, que desconsiderava os saberes da população e suas condições de vida. Os conteúdos tratados pelo modelo tradicional da EN abordavam os aspectos biológicos da alimentação, destacando o efeito dos nutrientes no organismo o que, embora seja uma informação importante, não considerava os aspectos regionais e as desigualdades sociais que comprometiam o acesso da população a uma alimentação adequada ${ }^{24}$.

A EN, quando praticada no âmbito dos programas de prevenção da DVA, também utilizava o modelo tradicional, desconsiderando os saberes tradicionais e as limitações de ordem econômica e social da população beneficiada. Esse fator, somado ao fato de ter sido praticada de forma irregular, não trouxe mudanças aos hábitos alimentares nas populações atingidas pela DVA.

O conceito de "Educação Nutricional Crítica", desenvolvido em 1984 por Valente ${ }^{25}$, e fruto das mudanças políticas do Brasil na época, permitiu uma evolução conceitual da EN, integrando vários aspectos que até então eram ignorados, como os políticos, ideológicos e econômicos, além de acesso a terra, emprego e renda, educação e serviços de saúde, entre outros, 
destacando-os como essenciais para a aquisição dos alimentos e a manutenção da saúde. Passava-se a discutir os determinantes da fome no país e a enfatizar que a EN, isoladamente, não poderia trazer alterações aos hábitos alimentares da população, sendo necessário, também, esclarecer as classes populares sobre os seus direitos de cidadão ${ }^{26}$.

O debate sobre Segurança Alimentar, iniciado em 1985, reforçado em 1986 pela I Conferência Nacional de Alimentação e Nutrição (um desdobramento da $8^{\circ}$ Conferência Nacional de Saúde) e a criação, em 1993, do Conselho Nacional de Segurança Alimentar e Nutricional (CONSEA), que organizou e coordenou a I Conferência Nacional de Segurança Alimentar, realizada em 1994, foi, também, um marco importante para a EN no Brasil. Esse é um aspecto que deve ser levado em consideração na prevenção da DVA, pois seu principal fator etiológico é a ausência de vitamina A na alimentação devido à insegurança alimentar da população atingida.

A discussão das diretrizes básicas de uma política nacional de segurança alimentar e nutricional, com representantes da sociedade civil e do governo, destaca a EN como um estímulo para as práticas alimentares e estilos de vida saudáveis ${ }^{28}$ e os programas de prevenção da DVA devem estar contemplados nessa política mais ampla.

A partir da década de 90 os fatos relatados tiveram influência na forma como a EN foi conduzida no âmbito dos programas oficiais de combate à DVA, trazendo uma mudança significativa, reforçada, também, pela publicação da Estratégia Global para Alimentação Saudável, Atividade Física e Saúde ${ }^{27}$, cujo objetivo principal é a promoção de hábitos de vida saudáveis e a prevenção e controle das doenças crônicas não-transmissíveis. Com isso, a EN passou a ser reconhecida como uma ação de promoção da saúde, sendo, portanto, mais valorizada e citada como estratégia de combate à DVA nos programas oficiais ${ }^{20}$.

Permanece o desafio de como desenvolver a prática da EN no País e, segundo Boog 23,29, inclui-se a promoção da alimentação saudável, valori- zando e resgatando a cultura alimentar, mas considerando, também, a segurança alimentar. Além de respeitar e modificar crenças e atitudes em relação à alimentação, é preciso levar em conta o acesso econômico e social à alimentação adequada, promover essa questão em programas comunitários e de saúde e discutir a fome, pobreza e desnutrição em todos os setores da sociedade. É preciso valorizar, portanto, além dos conhecimentos científicos e técnicos sobre alimentação, o saber original da comunidade, permitindo que ela, confrontada com o novo e com o que já dispõe, faça as suas próprias escolhas. Essa prática tem um diferencial significativo, mostrando que, quando a comunidade faz suas próprias escolhas a partir da reflexão do que Ihe foi transmitido, acontece uma verdadeira mudança nos hábitos alimentares, que passarão a fazer parte do seu cotidiano.

O aumento do consumo de legumes e verduras ${ }^{30}$, por exemplo, importante para a prevenção da DVA e uma prioridade da Política Nacional de Alimentação e Nutrição, deve fazer parte dos conteúdos de EN ministrados no diversos setores da sociedade. Alguns pontos da educação ambiental referentes ao uso de agrotóxicos, consumo adequado de água, destino de resíduos, entre outros, devem também estar contemplados na EN pela sua importância atual na qualidade de vida e da alimentação. Outras medidas paralelas, como o incentivo ao plantio em hortas domiciliares, escolares e comunitárias, o ensino de formas de preparação e consumo desses alimentos, a formação de cooperativas de pequenos agricultores e a organização de feiras locais, sem intermediários, para melhorar o preço dos produtos oferecidos, devem ser incentivadas. Além disso, as escolas devem priorizar o consumo de alimentos naturais e orgânicos em suas merendas, utilizando a horta escolar como um instrumento pedagógico interdisciplinar.

A mídia desempenha importante papel na EN por permitir veicular programas de conscientização nacional, esclarecendo a importância do consumo de frutas, verduras e legumes para a 
304 | L.P.F. RODRIGUES \& M.J. RONCADA

manutenção da saúde e incentivando ações comunitárias que fortaleçam as mensagens veiculadas, para que os participantes, de forma ativa, possam fazer suas escolhas alimentares.

\section{O N CLUS Ã O}

A partir do que foi relatado ao longo deste trabalho, conclui-se que as limitações ocorridas na área de EN nos programas oficiais de combate e prevenção à DVA, devem-se principalmente ao fato de as ações educativas não fazerem parte de um contexto mais amplo de promoção da saúde, o que interfere na transformação duradoura de maus hábitos alimentares das populações de risco.

O relato histórico das ações de EN nos programas oficiais de combate à DVA confirma esse fato, ao deixar claro que essas ações tendem a não se realizar ou sofreram interrupções, ocorrendo de forma temporária e sem avaliação, conforme pode ser constatado no Brasil, ao longo dos últimos quarenta anos.

O programa oficial de combate à DVA, atualmente em vigor, avançou nesse sentido, pois já não recomenda a EN como medida pontual, mas considera que, paralelamente à administração da vitamina A nas áreas de risco, seja priorizada uma política de promoção da saúde, enfatizando-se tanto a necessidade de melhorar os índices e duração do aleitamento materno quanto o incentivo à alimentação saudável.

Acredita-se que, à medida que a EN avance e se estabeleça como parte de uma política pública intersetorial de promoção para a saúde, haja uma evolução dos conteúdos e metodologias propostos, que serão pesquisados e incorporados à sua prática, promovendo a alimentação saudável e prevenindo as doenças carenciais, entre elas a DVA, bem como as doenças crônicas não transmissíveis.

Recomenda-se, portanto, que os vários setores da sociedade, públicos e privados, se envolvam com o tema, debatendo as principais dificuldades, propondo ações conjuntas, pesquisando novas metodologias, envolvendo sujeitos e comunidades, para que ocorra uma transformação duradoura dos valores que impedem o consumo de uma alimentação saudável.

A atuação do profissional Nutricionista, responsável principal pela prática da EN, se amplia com a intersetorialidade, indo muito além das unidades de saúde. No entanto, para exercer seu papel de Educador, disciplinas como Antropologia, Educação Ambiental, Pedagogia e Sociologia devem estar contempladas na grade curricular dos Cursos de Nutrição do Brasil, permitindo a formação de profissionais mais bem preparados e conscientes para o exercício da EN inserida como medida de promoção da saúde.

\section{COLABORADORES}

L.P.F. RODRIGUES e M.J. RONCADA participaram em todas as fases da pesquisa.

\section{REFER Ê N CIAS}

1. World Health Organization. The global prevalence of vitamin a deficiency. Micronutrient Deficiency Information System (MDIS). Geneva: WHO;1995.

2. Diniz AS Santos LMP. Hipovitaminose A e xeroftalmia. J Pediatr (Rio de Janeiro). 2000; 76(3):311-22. doi: 10.1590/S1519-38292007000100008.

3. Dolinsky $M$, Ramalho RA. Deficiência de vitamina A: uma revisão atualizada. Comp Nutr. 2003; 2(4): 2-17.

4. Geraldo RRC, Paiva SAR, Pitas AMCS, Godoy I, Campana A. Distribuição da hipovitaminose A no Brasil nas últimas décadas: ingestão alimentar, sinais clínicos e dados bioquímicos. Rev Nutr. 2003; 4(16):443-60. doi: 10.1590/S1415-5273200300 0400008.

5. Milagres RCRM, Nunes LC, Pinheiro-Sant'ana HM. A deficiência de vitamina A em crianças no Brasil e no mundo. Ciênc Saúde Colet. 2007; 5(12): 1253-66. doi: 10.1590/S1413-8123200700050 0023.

6. Ramalho RA, Flores $H$, Saunders $C$. Hipovitaminose A no Brasil: um problema de saúde pública. Pan Am J Public Health. 2002; 2(12):117-22.

7. Souza WA, Villas Boas OMGC. A deficiência de vitamina A no Brasil: um panorama. Rev Panam Salud Publica. 2002; (12)3:173-9. 
8. Brasil. Ministério da Saúde. Carências de micronutrientes. Brasília: MS; 2007. Cadernos de Atenção Básica, 20.

9. Brasil. Ministério da Saúde. Programa Nacional de Suplementação de Vitamina A. Brasília: Instituto Nacional de Alimentação e Nutrição; 1994.

10. Roncada MJ. Vitaminas lipossolúveis. In: Dutra de Oliveira JE, Marchini JS. Ciências nutricionais. São Paulo: Sarvier; 2000.

11. Gil AC. Métodos e técnicas de pesquisa social. São Paulo: Atlas; 2007.

12. Araújo RL. Situação alimentar e nutricional do Brasil. Brasília: Tipogresso;1989.

13. Brasil. Ministério da Saúde. Hipovitaminose A no Brasil. Documento Básico. Brasília: Instituto Nacional de Alimentação e Nutrição; 1977.

14. Lobato A. Projetos e programas para o controle da hipovitaminose A no Brasil. Brasília: Ministério da Saúde; 1998.

15. Brasil. Ministério da Saúde. Combate à Hipovitaminose A no Brasil. Projeto Preliminar. Brasília: Instituto Nacional de Alimentação e Nutrição; 1981.

16. Organização Mundial de Saúde. Vitamina A na gestação e lactação: recomendações e relatório de uma consultoria. Recife: Centro Colaborador de Alimentação e Nutrição do Nordeste; 2001.

17. Martins MC, Oliveira YP, Coitinho DC, Santos LMP. Panorama das ações de controle da deficiência de vitamina A no Brasil. Rev Nutr. 2007; 20(1):5-18. doi: 10.1590/S1415-52732007000100001.

18. Brasil. Ministério da Saúde. Cartilha: conheça a hipovitaminose A e aprenda a preveni-la. Brasília: Instituto Nacional de Alimentação e Nutrição; 1994.

19. Brasil. Ministério da Saúde. Projeto Suplementação de megadoses de vitamina A no pós-parto imediato nas maternidades/hospitais. Brasília: Secretaria de Políticas de Saúde; 2002.

20. Brasil. Ministério da Saúde. Vitamina A Mais: programa nacional de suplementação vitamina A. Brasília: Secretaria de Políticas de Saúde; 2004.
21. Martins MC, Santos LMP, Santos SMC, Araújo MPN, Lima AMP, Santana LAA. Avaliação de políticas públicas de segurança alimentar e combate à fome no período 1995-2002. Cad Saúde Pública. 2007; (23)9:2081-93. doi: 101590/ S0102-311X2007009 0016.

22. Levy SN, Silva JJC, Cardoso IFR, Werberich PM, Montiani H, Carneiro RM. Educação em saúde: histórico, conceitos e propostas. Brasília: Ministério da Saúde. [Internet] [acesso 2008 set 20]. Disponível em: <http://www.datasus.gov.br/cns/data sus.htm>.

23. Boog MCF. Educação nutricional em serviços públicos de saúde. Cad Saúde Publica. 1999; 15(Sup.2): 139-47. doi: 10.1590/S0102-311X1999000600 014.

24. Ferreira VA, Magalhães, R. Nutrição e promoção da saúde: perspectivas atuais. Cad Saúde Pública. 2007; (23)7:1674-81. doi: 10.1590/S0102-311X2 007000700019.

25. Valente JC. Em busca de uma educação nutricional crítica. In: Fome e desnutrição, determinantes sociais. São Paulo: Cortez;1986.

26. Santos LAS. Educação alimentar e nutricional no contexto da promoção de práticas alimentares saudáveis. Rev Nutr. 2005; 18(5):681-92.

27. Valente JC. Direito humano à alimentação: desafios e conquistas. São Paulo: Cortez; 2002.

28. Organização Mundial de Saúde. Estratégia global para alimentação saudável, atividade física e saúde. Genebra: OMS; 2004.

29. Boog MCF. Educação nutricional: passado, presente e futuro. Rev Nutr. 1997 10(1):5-19.

30. Brasil. Ministério da Saúde. A Iniciativa de incentivo ao consumo de frutas, legumes e verduras $(F, L \& V)$ no Brasil: documento base. Brasília: Ministério da Saúde; 2004.

Recebido em: 7/12/2007

Versão final reapresentada em: 23/7/2009 Aprovado em: 11/11/2009 
